\title{
Concurrent Validity of the ABC Dementia Scale with Other Standard Scales: A New Comprehensive Instrument for Assessing Dementia in Japan
}

\author{
Wataru Shimoda $^{a}$ Jun Murata $^{\mathrm{b}}$ Akira Nakatani $^{\mathrm{c}}$ Katsuya Satoh $^{\mathrm{a}}$ \\ aDepartment of Locomotive Rehabilitation Science, Nagasaki University Graduate School of Biomedical Sciences, \\ Nagasaki, Japan; 'Department of Physical and Occupational Therapy, Graduate School of Biomedical Sciences, \\ Nagasaki, Japan; ${ }^{\text {Y }}$ amanote Clinic, Nagasaki, Japan
}

\section{Keywords}

Neuropsychological tests · Alzheimer disease · Vascular dementia $\cdot A B C$ Dementia Scale

\begin{abstract}
Background/Aims: The $A B C$ Dementia Scale (ABC-DS), a new tool for evaluating dementia, was developed in Japan. The ABC-DS is a comprehensive instrument that can simultaneously evaluate activities of daily living (ADLs), behavioral and psychological symptoms of dementia (BPSD), and cognitive function. The ABC-DS can be administered easily and quickly and can clarify the severity of dementia and its changes over time. While the ABC-DS has been reported to be useful in Alzheimer disease (AD)-type dementia, it has not yet been studied in other types of dementia. The purpose of this study was to reevaluate the standard validity of $A B C-D S$ separately for various dementia types and severities. Methods: We evaluated the ABC-DS in outpatients at 1 hospital in Nagasaki Prefecture and patients who use the facility. Domain $A$, corresponding to ADLs, correlated with Disability Assessment for Dementia (DAD); domain B, corresponding to BPSD, correlated with the Neuropsychiatric Inventory (NPI); domain $\mathrm{C}$, corresponding to cognitive functions, correlated with Mini-Mental State Examination (MMSE); and the total
\end{abstract}

score of the ABC-DS correlated with the Clinical Dementia Rating (CDR). Results: 102 patients, comprising 38 males and 64 females with an average age of $80.7 \pm 8.6$ years, were enrolled. AD-type dementia was present in 38 cases, vascular dementia (VaD) in 23, mixed dementia in 23, dementia with Lewy bodies in 6, argyrophilic grain dementia in 9, and mild cognitive impairment in 3. A strong correlation was found between $A B C-D S$ domain scores and their respective reference neuropsychological instruments (domain $A$ and the $D A D$, domain $B$ and the $\mathrm{NPI}$, domain $C$ and the MMSE, and total score and (DR). The correlation of each ABC-DS domain score with the corresponding standard scale depended on the type and severity of dementia, and we observed moderate or high correlations in $\mathrm{AD}$ and $\mathrm{VaD}$ patients with moderate and severe dementia. Discussion: Although the ABC-DS targets $A D$, it can be used in $V a D$ based on the results of this study. In other types of dementia, the results differed depending on the domain; in some conditions, the ABC-DS may not show sufficient concurrent validity with other standard scales. Also, the ABC-DS is more beneficial for moderate-to-severe dementia, as reported in previous studies. It is highly useful in clinical practice in Japan since there more than half of all patients have moderate-to-severe dementia.

(c) 2021 The Author(s)

Published by S. Karger AG, Basel karger@karger.com www.karger.com/dem

Karger $\stackrel{2}{*}$

BOPEN ACCESS
(C) 2021 The Author(s)

Published by S. Karger AG, Basel

This is an Open Access article licensed under the Creative Commons Attribution-NonCommercial-4.0 International License (CC BY-NC) (http://www.karger.com/Services/OpenAccessLicense), applicable to the online version of the article only. Usage and distribution for commercial purposes requires written permission.
Katsuya Satoh

Department of Locomotive Rehabilitation Science, Nagasaki University Graduate Schoo of Biomedical Sciences, Unit of Rehabilitation Sciences

1-7-1, Sakamoto Town, Nagasaki City 852-8520 (Japan) satoh-prion@ nagasaki-u.ac.jp 


\section{Introduction}

With the rapid aging of the population in Japan, the numbers of patients with dementia will increase in the coming years. It is estimated that the number of elderly patients with dementia will rise from 4.62 million in 2012 to 7.3 million in 2025, at which time approximately 1 in 5 individuals aged 65 years or older will be affected by this disease [1].

Hasegawa's Dementia Scale-Revised (HDS-R) [2, 3] and the Mini-Mental State Examination (MMSE) [4] are commonly used psychometric tests in Japan for screening patients with cognitive impairment. These questionnaires primarily cover the examinee's orientation to time and space, arithmetic ability, short-term memory, and delayed recall. Home-based care and support are key components of the Japanese Government's welfare policy for patients with dementia. In real-life situations, however, these are not practical solutions because of decreases in activities of daily living (ADLs) and onset of behavioral and psychological symptoms of dementia (BPSD). In clinical settings, the Disability Assessment for Dementia (DAD) [5] and Neuropsychiatric Inventory (NPI) [6] questionnaires are often used for the assessment of ADLs and BPSD, respectively. However, each of these tools requires around $30 \mathrm{~min}$ to administer, and they fail to provide an overall picture of the respondent's condition.

The Clinical Dementia Rating (CDR) scale [7] was developed to characterize 6 domains of cognitive and functional performance of patients with dementia: memory, orientation, judgment and problem solving, community affairs, home and hobbies, and personal care. However, this tool has not been widely adopted in Japan because it requires a patient interview and a reliable informant (e.g., family member) and takes approximately $1 \mathrm{~h}$ to complete. With the exception of the HDS-R, the psychometric assessment instruments mentioned above were originally developed in Western countries, and their cross-cultural adaptation remains a challenge. Against this background, we needed a new, easy-to-administer, comprehensive tool for the assessment and screening of dementia patients in Japan. Specifically, we aimed for this test to be relevant to the Japanese cultural context and to cover ADLs, BPSD, and cognitive functions.

The ABC Dementia Scale (ABC-DS) is a 13-item, interview-based, multidimensional questionnaire to evaluate the severity of Alzheimer's disease (AD), first developed in 2016 by a group of neuropsychiatrists, neurologists, and geriatricians in Japan [8-10]. The acronym
"ABC" refers to ADLs, BPSD, and cognitive functions. This instrument allows for the assessment of each of these domains (A, B, and C) and overall condition (as a sum of the 3 domain scores). This scale is useful for screening patients with moderate and severe $\mathrm{AD}$ and for monitoring disease progression. The ABC-DS questionnaire can be completed in approximately $10 \mathrm{~min}$ and categorizes disease severity in a manner similar to the CDR scale. Moreover, the ABC-DS test can be administered by medical specialists, general practitioners, and other healthcare professionals with no or little prior training. This instrument is gaining popularity in Japan.

The Finnish Geriatric Intervention Study to Prevent Cognitive Impairment and Disability (FINGER) trial and other studies evaluated the effects of multidomain lifestyle intervention among elderly at-risk individuals and showed that cognitive decline can be prevented with improvements in executive function, processing speed, and memory tasks [11-13]. To evaluate the impact of multimodal intervention in older people, a global assessment tool is needed to assess cognitive functions and disease progression.

We conducted a preliminary study to evaluate the utility of the ABC-DS test in residents of dementia care facilities, but the results did not support the validity of the instrument in patients with non-AD dementia. The study population of the previous study by Mori et al. [11] consisted of patients with moderate and severe dementia [10], and few studies to date have administered the ABCDS to individuals with mild cognitive impairment or mild dementia. The present study was designed and implemented by taking into account the challenges identified in the aforementioned previous studies.

\section{Methods}

Subjects

Seniors who were receiving dementia care services at outpatient hospitals and residential dementia care facilities located in Nagasaki Prefecture, Japan, and who provided informed consent to participate, were eligible for inclusion. Exclusion criteria included withdrawal of consent, onset of delirium, and sudden worsening of BPSD.

Participants were recruited at 2 clinics in Japan. Those who were enrolled had been diagnosed with (1) AD based on the criteria of the Diagnostic and Statistical Manual of Mental Disorders, Fourth Edition, text revision (DSM-V) [14] and the International Classification of Diseases (ICD)-10; (2) probable AD based on the criteria of either the National Institute on AgingAlzheimer's Association (NIA-AA) workgroups [15] or the National Institute of Neurological and Communicative Disorders and Stroke and the Alzheimer's Disease and Related Disorders 
Association [16]; or (3) mild cognitive Impairment based on the DSM-V or NIA-AA diagnostic criteria [17]. We also enrolled patients diagnosed with vascular dementia $(\mathrm{VaD})$ based on diagnostic and classification systems including the ICD-10 and DSM-V [14] and those diagnosed with dementia with Lewy bodies (DLB) [18].

\section{Assessment}

Occupational therapists and physicians who agreed to take part in this study conducted a semi-structured interview and administered the ABC-DS to the informants of study participants (professional care workers or family members). For each study participant, scores for domain $\mathrm{A}$, domain $\mathrm{B}$, domain $\mathrm{C}$, and the total ABC-DS were determined. The DAD, NPI, MMSE, and CDR-SOB were evaluated as reference tests for domain $\mathrm{A}$, domain $\mathrm{B}$, domain $\mathrm{C}$, and total scores, respectively. The severity score of the NPI (on a 1-3 scale) was used for comparison with domain B. Moreover, the effectiveness of the total ABC-DS score as an indicator for the global CDR score was analyzed using the receiver operating characteristic (ROC) analysis.

\section{Three-Dimensional Distance}

We calculated the mathematical distance of the scores (ADL score, BPSD score, and cognitive function score) from the origin $(0,0,0)$ and used this distance as a total score. The three-dimensional distance (TDD) score was calculated as follows: TDD = $\sqrt{ }\left((\text { Domain } \mathrm{A})^{2}+(\text { Domain } \mathrm{B})^{2}+(\text { Domain } \mathrm{C})^{2}\right)$, where the ADL, $\mathrm{BPSD}$, and cognitive function scores increase with symptom improvement and decrease toward 0 with symptom deterioration. The difference over time was thus -1.8 , indicating disease progression. An international patent application has been made for the concept of the TDD [19].

\section{Statistical Analysis}

The criterion-referenced validity of the 3 domains and total ABC-DS scale was evaluated by analyzing correlations with their respective tests. The correlation between the total ABC-DS score and global CDR score was assessed based on the Spearman's rank correlation coefficient. In addition, ROC curves were used to establish cutoff points that maximize sensitivity and specificity of the total ABC-DS score. For the $3 \mathrm{ABC}$-DS domains, correlations were assessed based on Pearson's correlation coefficient. Statistical analysis was performed using IBM SPSS ${ }^{\circledR}$ Statistics version 22 (IBM Japan, Tokyo).

\section{Ethical Considerations}

This study was designed to include patients with dementia whose capability or competency to give formal consent might be compromised. Therefore, dementia patients with an HDS-R score $\leq 15$ were classified as ineligible for formal consent, and their legal guardians were asked to provide written informed consent for this study.

During the informed consent process, the investigators explained to prospective study participants or their legal guardians the purpose and procedures of the study, freedom to refuse study participation without any loss of benefits, liberty to discontinue at any time, and protection of their privacy and confidentiality. Before its start, this study was approved by the Ethics Committee of the Nagasaki University Graduate School of Biomedical Sciences (No. 19071101).

Validity of ABC-DS
Table 1. Subject characteristics and baseline data

$\begin{array}{lc}\text { Overall population } & \\ \text { Subjects, } N & 102 \\ \text { Age, years } & 83.1 \pm 7.9 \\ \text { Gender, M/F } & 38 / 64\end{array}$

Disease types, $n$

AD-type dementia $\quad 38$

Vascular dementia $\quad 23$

Mixed dementia $\quad 23$

Dementia with Lewy bodies 6

Argyrophilic grain disease 9

Mild cognitive impairment 3

CDR severity, $n$

CDR 1

CDR $2 \quad 39$

CDR 3

Type of care, $n$

Outpatient hospital $\quad 81$

Dementia facility $\quad 21$

AD, Alzheimer's disease; CDR, Clinical Dementia Rating.

\section{Results}

\section{Subjects Analysis}

This study included 102 participants (38 males and 64 females) with a mean age of 83.1 (standard deviation [SD] 7.9) years. Of these, 81 and 21 received care services in outpatient hospital and residential dementia care facility settings, respectively. By diagnosis, 38, 23, $23,6,9$, and 3 participants had $\mathrm{AD}$-type dementia, $\mathrm{VaD}$, mixed dementia, DLB, argyrophilic grain disease, and mild cognitive impairment, respectively. The participants' demographic characteristics are summarized in Table 1.

The mean total ABC-DS score was $64.6 \pm 25.2$ (range $0-117)$, with mean domain $A$, domain $B$, and domain $C$ scores of $30.1 \pm 12.7$ (range $0-54$ ), $15.8 \pm 7.0$ (range $0-27$ ), and $18.7 \pm 10.0$ (range $0-36$ ), respectively. The mean DAD, NPI severity, MMSE, and CDR-SOB scores were $39.4 \pm 18.7,6.8 \pm 4.1,14.1 \pm 6.4$, and $12.6 \pm 4.2$, respectively. The score results are summarized in $\mathrm{Ta}$ ble 2 .

\section{Correlation Analysis}

Correlations between the ABC-DS domain and total scores and their respective reference test scores were analyzed. 


\section{Overall Population}

Strongly significant correlations were observed between domain A and the DAD score $(r=0.779, p<0.01)$, between domain B and the NPI severity score $(r=-0.713$, $p<0.01)$, and between domain $C$ and the MMSE score $(r=0.735, p<0.01)$ (Fig. 1). A moderately significant correlation was found between the total ABC-DS score and the CDR-SOB score $(r=-0.648, p<0.01)$. The results are shown in Table 3.

\section{Disease Types}

\section{AD-Type Dementia}

In 38 participants with $\mathrm{AD}$-type dementia, domain $\mathrm{A}$ $(r=0.841, p<0.01)$, domain B $(r=-0.788, p<0.01)$, and domain $\mathrm{C}(r=0.870, p<0.01)$ demonstrated strongly significant correlations with their respective tests. A moderately significant correlation was noted between the total ABC-DS score and the CDR-SOB score $(r=-0.762, p<$ 0.01 ) (Table 2; Fig. 2; see online suppl. Table 1; see www. karger.com/doi/10.1159/000513485 for all online suppl. material).

\section{$V a D$}

In 23 participants with $\mathrm{VaD}$, domain $\mathrm{A}(r=0.509, p<$ $0.05)$, domain $\mathrm{B}(r=-0.646, p<0.01)$, domain $\mathrm{C}(r=$ $0.444, p<0.05)$, and the total ABC-DS score $(r=-0.469$, $p<0.05)$ demonstrated moderately significant correlations with their respective reference tests (Fig. 3).

\section{Mixed Dementia}

In 23 participants with mixed dementia, domain $\mathrm{B}$ $(r=-0.619, p<0.05)$ and domain $\mathrm{C}(r=0.638, p<0.01)$ demonstrated strongly significant correlations with their respective reference tests. Neither domain A $(r=0.352$, $p=0.100)$ nor total ABC-DS score $(r=-0.206, p=0.345)$ exhibited a significant correlation with the respective reference test.

\section{Dementia with Lewy Bodies}

In 6 participants with DLB, a strongly significant correlation was found between domain $\mathrm{A}$ and its reference test $(r=0.835, p<0.05)$. However, domain $\mathrm{B}(r=0.145$, $p=0.785)$, domain $\mathrm{C}(r=-0.011, p=0.984)$, and the total ABC-DS score $(r=0.000, p=1.000)$ demonstrated no significant correlations with their respective reference tests.
Table 2. Correlation coefficients between the ABC-DS and respective reference tests (disease types)

\begin{tabular}{lrrrc}
\hline & $\begin{array}{c}\text { Corre- } \\
\text { lation }\end{array}$ & Lower & Upper & $\begin{array}{l}p \\
\text { value }\end{array}$ \\
\hline Domain A versus DAD & & & & \\
Overall population & 0.799 & 0.689 & 0.845 & $<0.01$ \\
AD-type dementia & 0.841 & 0.713 & 0.915 & $<0.01$ \\
VaD & 0.509 & 0.123 & 0.761 & $<0.05$ \\
DLB & 0.835 & 0.073 & 0.981 & $<0.05$ \\
Mixed dementia & 0.352 & -0.07 & 0.667 & 0.100 \\
Argyrophilic grain disease & 0.943 & 0.746 & 0.988 & $<0.01$ \\
Domain B versus NPI & & & & \\
Overall population & -0.713 & -0.797 & -0.602 & $<0.01$ \\
AD-type dementia & -0.788 & -0.885 & -0.626 & $<0.01$ \\
VaD & -0.646 & -0.836 & -0.319 & $<0.01$ \\
DLB & 0.145 & -0.755 & 0.856 & 0.785 \\
Mixed dementia & -0.619 & -0.822 & -0.278 & $<0.05$ \\
Argyrophilic grain disease & -0.382 & -0.834 & 0.378 & 0.311 \\
Domain C versus MMSE & & & & \\
Overall population & 0.735 & 0.635 & 0.815 & $<0.01$ \\
AD-type dementia & 0.870 & 0.774 & 0.935 & $<0.01$ \\
VaD & 0.444 & 0.039 & 0.724 & $<0.01$ \\
DLB & -0.011 & -0.815 & 0.808 & 0.384 \\
Mixed dementia & 0.638 & 0.306 & 0.832 & $<0.01$ \\
Argyrophilic grain disease & 0.909 & 0.618 & 0.981 & $<0.01$ \\
Total ABC-DS score versus & CDR-SOB & & & \\
Overall population & -0.648 & -0.748 & -0.519 & $<0.01$ \\
AD-type dementia & -0.762 & -0.870 & -0.585 & $<0.01$ \\
VaD & -0.469 & -0.738 & -0.070 & $<0.05$ \\
DLB & 0.000 & -0.812 & 0.812 & 1.000 \\
Mixed dementia & -0.206 & -0.570 & 0.225 & 0.345 \\
Argyrophilic grain disease & -0.593 & -0.902 & -0.117 & 0.092 \\
TDD versus CDR-SOB* & & & & \\
Overall population & -0.667 & -0.763 & -0.543 & $<0.01$ \\
AD-type dementia & -0.778 & -0.879 & -0.610 & $<0.01$ \\
VaD & -0.641 & -0.833 & -0.311 & $<0.01$ \\
DLB & -0.203 & -0.872 & 0.729 & 0.700 \\
Mixed dementia & -0.196 & -0.563 & 0.235 & 0.370 \\
Argyrophilic grain disease & -0.707 & -0.933 & -0.081 & $<0.05$ \\
\hline & & & & \\
& & & & \\
& & & & \\
& & & & \\
& & & & \\
& & &
\end{tabular}

ABC-DS, ABC Dementia Scale; DAD, Disability Assessment for Dementia; $\mathrm{AD}$, Alzheimer's disease; $\mathrm{VaD}$, vascular dementia; DLB, dementia with Lewy bodies; NPI, Neuropsychiatric Inventory; MMSE, Mini-Mental State Examination; CDR, Clinical Dementia Rating. * CDR-SOB: total sum of box scores in the CDR.

\section{Argyrophilic Grain Disease}

In 9 participants with argyrophilic grain disease, domain A $(r=0.943, p<0.01)$ and domain C $(r=0.909, p<$ $0.05)$ demonstrated strongly significant correlations with their respective reference tests. No significant correlation was observed between domain B and NPI severity $(r=$ $-0.382, p=0.311)$. No statistical analysis was conducted for the relationship between the total ABC-DS score and 

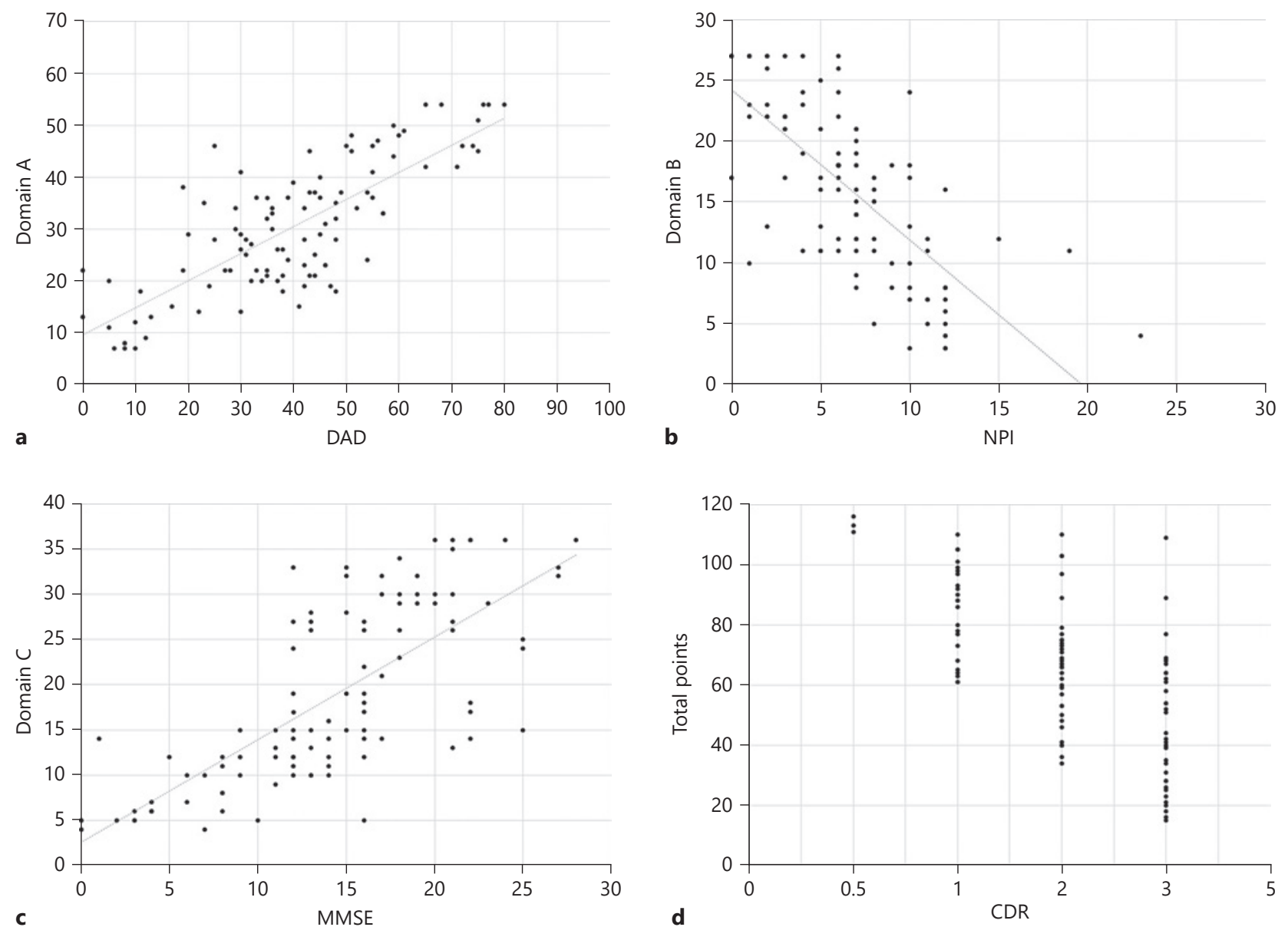

Fig. 1. The relationship between $A B C-D S$ score and their respective reference neuropsychological instruments in all cases. The relationship between domain A of ABC-DS and DAD (correlation coefficient: $\left.R^{2}=0.779\right)(\mathbf{a})$, the relationship between domain B of ABC-DS and NPI (correlation coefficient: $\left.R^{2}=0.7139\right)(\mathbf{b})$, the relationship between domain C of ABC-DS and MMSE (correlation coefficient: $\left.R^{2}=0.735\right)(\mathbf{c}$ ), and the relationship between total scores of ABC-DS and CDR (correlation coefficient: $\left.R^{2}=0.629\right)(\mathbf{d})$. ABC-DS, ABC Dementia Scale; DAD, Disability Assessment for Dementia; NPI, Neuropsychiatric Inventory; MMSE, Mini-Mental State Examination; CDR, Clinical Dementia Rating.

the global CDR score because all 9 participants in this subgroup had a global CDR score of 3, but the relationship between the total ABC-DS score and the CDR-SOB score was moderate $(r=0.593, p<0.092)$.

\section{CDR Severity}

\section{CDR 1 (Mild Stage)}

Twenty-three participants had mild dementia with a global CDR score of 1 . In these participants, a moderately significant correlation was observed between domain
A and the DAD score $(r=0.522, p<0.05)$, whereas neither domain $\mathrm{B}(r=-0.380, p=0.074)$ nor domain $\mathrm{C}(r=0.251$, $p=0.248$ ) demonstrated a significant correlation with the respective reference test. Neither TDD nor the total ABC score showed a significant correlation with the CDR-SOB score $([r=-0.325, p=0.131]$ and $[r=-0.252, p=0.246]$, respectively) (Table 4 ).

\section{CDR 2 (Moderate Stage)}

Thirty-nine participants had moderate dementia with a global CDR score of 2 . In these participants, a moderately significant correlation was observed between do- 
main A and the DAD score $(r=0.503, p<0.05)$. Meanwhile, domain $\mathrm{B}(r=-0.741, p<0.01)$ and domain $\mathrm{C}(r=$ $0.770, p<0.01)$ demonstrated strongly significant correlations with their respective reference tests. Neither TDD nor the total $\mathrm{ABC}$ score showed a significant correlation with the CDR-SOB score $([r=-0.159, p=0.334]$ and $[r=-0.146, p=0.374]$, respectively).

\section{CDR 3 (Severe Stage)}

Thirty-seven participants had severe dementia with a global CDR score of 3 . In these participants, domain A $(r=0.767, p<0.01)$ and domain $\mathrm{C}(r=0.790, p<0.01)$ demonstrated strongly significant correlations with their respective reference tests. In addition, a moderately significant correlation was observed between domain $\mathrm{B}$ and NPI severity $(r=-0.593, p<0.01)$. The total ABC score and TDD both demonstrated the same correlation with the CDR-SOB score $(r=-0.334, p<0.05)$.

\section{Type of Care}

\section{Outpatient Hospital (Family Home Care)}

Eighty-one participants received dementia care services in the outpatient hospital (family home care) setting. In these participants, domain A $(r=0.843, p<0.01)$, domain $\mathrm{B}(r=-0.792, p<0.01)$, and domain $\mathrm{C}(r=0.824$, $p<0.01)$ demonstrated strongly significant correlations with their respective reference tests. In addition, a moderately significant correlation was found between the total ABC-DS score and the CDR-SOB score $(r=-0.638$, $p<0.01$ ) (Table 5; online suppl. Table 2).

\section{Dementia Facility (Professional Home Care)}

Twenty-one participants received dementia care services in a residential dementia care (professional home care) facility setting. Correlations with respective reference tests were strongly significant for domain A $(r=$ $0.793, p<0.01)$ and the total ABC-DS score $(r=-0.813$, $p<0.01)$, and moderately significant for domain $\mathrm{B}(r=$ $-0.663, p<0.05)$ and domain $\mathrm{C}(r=0.627, p<0.05)$.

\section{Disease Severity}

ROC analysis was conducted to determine the cutoff score of the total ABC-DS as a predictor for the global CDR score. An ABC-DS cutoff score of 76.0 points differentiated between a global CDR score range of $0.5-1$ and a range of $2-3$ (sensitivity 0.66 , specificity 0.90 ). In addition, an ABC-DS cutoff score of 58.5 points differentiated between a global CDR score of 3 and a range of $0.5-2$, with
Table 3. Correlation coefficients between the ABC-DS and respective reference tests in the overall patient samples (CDR severity)

\begin{tabular}{lcccc}
\hline & $\begin{array}{c}\text { Corre- } \\
\text { lation }\end{array}$ & Lower & Upper & $p$ value \\
& & & & \\
\hline Domain A versus DAD & 0.522 & 0.14 & 0.769 & $<0.05$ \\
CDR 1 (mild) & 0.503 & 0.223 & 0.706 & $<0.05$ \\
CDR 2 (moderate) & 0.767 & 0.589 & 0.874 & $<0.01$ \\
CDR 3 (severe) & & & & \\
Domain B versus NPI & -0.38 & -0.685 & 0.038 & 0.074 \\
CDR 1 (mild) & -0.741 & -0.856 & -0.555 & $<0.01$ \\
CDR 2 (moderate) & -0.593 & -0.769 & -0.333 & $<0.01$ \\
CDR 3 (severe) & & & & \\
Domain C versus MMSE & 0.251 & -0.18 & 0.601 & 0.248 \\
CDR 1 (mild) & 0.767 & 0.596 & 0.872 & $<0.01$ \\
CDR 2 (moderate) & 0.790 & 0.626 & 0.887 & $<0.01$ \\
CDR 3 (severe) & &
\end{tabular}

ABC-DS, ABC Dementia Scale; CDR, Clinical Dementia Rating; DAD, Disability Assessment for Dementia; NPI, Neuropsychiatric Inventory; MMSE, Mini-Mental State Examination.

a sensitivity of 0.82 and a specificity of 0.68 . The areas under the ROC curves for the ABC-DS cutoff scores of 76.0 and 58.5 points were 0.85 and 0.82 , respectively.

As mentioned above, we calculated the TDD score for each patient. We also added to the total sum of the box index in the CDR score (CDR-SOB). $\mathrm{AD}$ and $\mathrm{VaD}$ showed a strong relationship between the TDD and CDR-SOB scores. Thus, the stages of $\mathrm{AD}$ and $\mathrm{VaD}$ are associated with the severity as the TDD of the ABC-DS represents severity.

\section{Discussion}

The criterion-referenced validity of the ABC-DS scale was evaluated by analyzing correlations with reference tests. In the overall study population, domain A, domain $\mathrm{B}$, domain $\mathrm{C}$, and the total ABC-DS score showed moderate or strong correlations with their respective reference tests. However, subgroup analyses by disease type and severity showed several differences worthy of note.

Whereas previous studies of the ABC-DS questionnaire evaluated $\mathrm{AD}$, the primary disease of interest for this instrument, this study included other types of dementia to investigate whether the instrument had broader applicability. Patients with AD showed similar results to the overall study population, providing supporting evidence for 


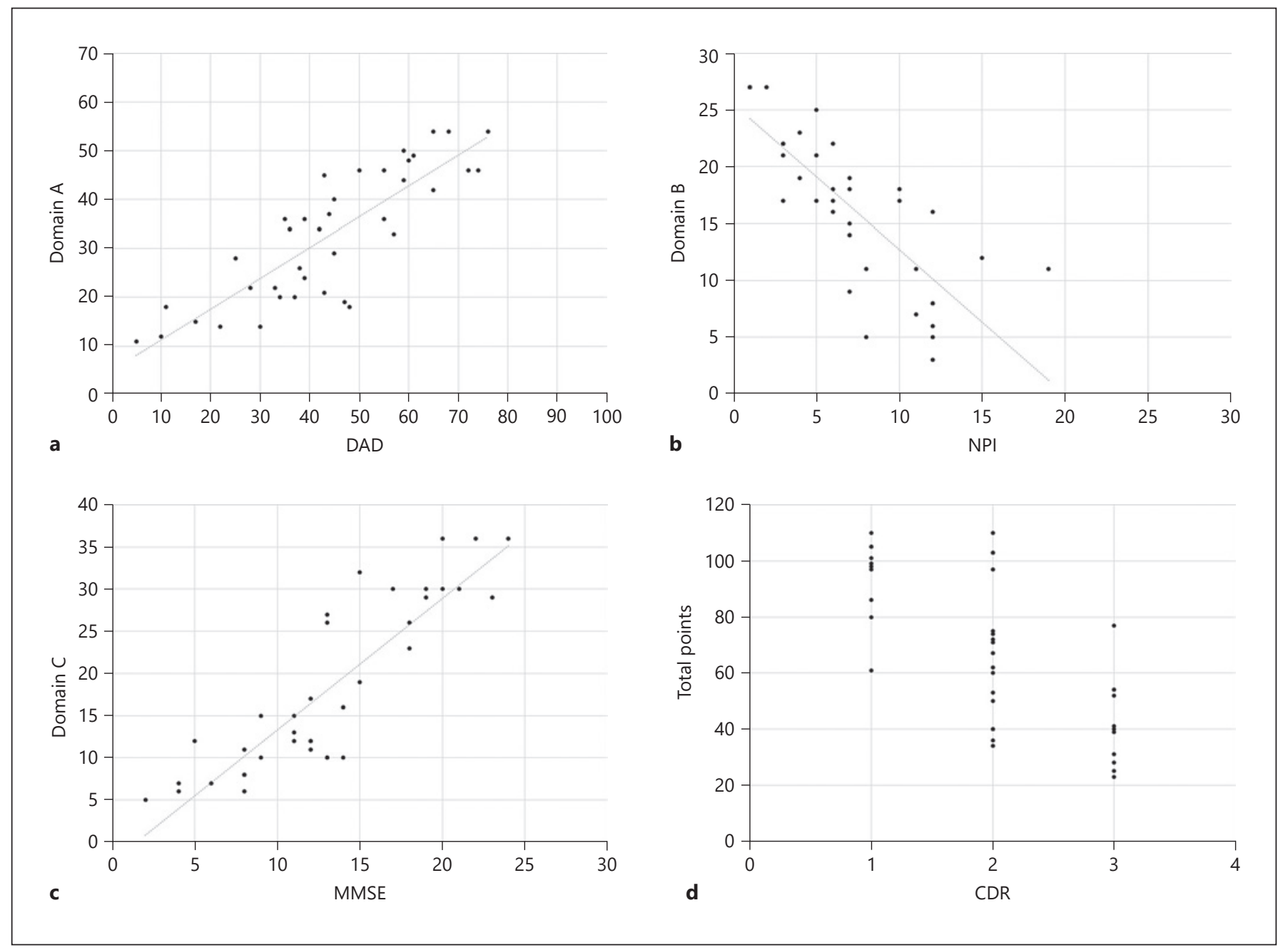

Fig. 2. The relationship between ABC-DS score and their respective reference neuropsychological instruments in patients with dementia of Alzheimer's disease-type dementia. The relationship between domain A of ABC-DS and DAD (correlation coefficient: $\left.R^{2}=0.841\right)(\mathbf{a})$, the relationship between domain $\mathrm{B}$ of ABC-DS and NPI (correlation coefficient: $\left.R^{2}=0.788\right)(\mathbf{b})$, the relationship be- tween domain C of ABC-DS and MMSE (correlation coefficient: $\left.R^{2}=0.870\right)(\mathbf{c})$, and the relationship between total scores of ABCDS and CDR (correlation coefficient: $R^{2}=0.688$ ) (d). ABC-DS, ABC Dementia Scale; DAD, Disability Assessment for Dementia; NPI, Neuropsychiatric Inventory; MMSE, Mini-Mental State Examination; CDR, Clinical Dementia Rating. prior studies. In patients with $\mathrm{VaD}$, domain $\mathrm{A}$, domain $\mathrm{B}$, domain $\mathrm{C}$, and the total $\mathrm{ABC}$-DS score showed moderate correlations with their respective reference tests. These results suggest the utility of the scale for this type of dementia. For mixed dementia, DLB, and argyrophilic grain disease, several domains showed strong correlations, suggesting the scale's possible (but limited) utility.

DAD, NPI, CDR, and MMSE have been used to evaluate $\mathrm{ADL}, \mathrm{BPSD}$, and cognitive function in various dementias, as well as in diseases that cause dementia symptoms, whereas DLB and FTLD are disease-specific evalu- ation scales. DAD, NPI, CDR, and MMSE are utilized frequently [20-22].

Since previous studies of the ABC-DS scale focused on patients with moderate-to-severe dementia, its utility for the assessment of patients with mild cognitive impairment and mild dementia remained elusive. Our results for different disease severities showed moderate or strong correlations between the $3 \mathrm{ABC}$-DS domains and their respective reference tests in patients with global CDR scores of 2 (moderate dementia) and 3 (severe dementia). However, only domain A showed a significant correlation 


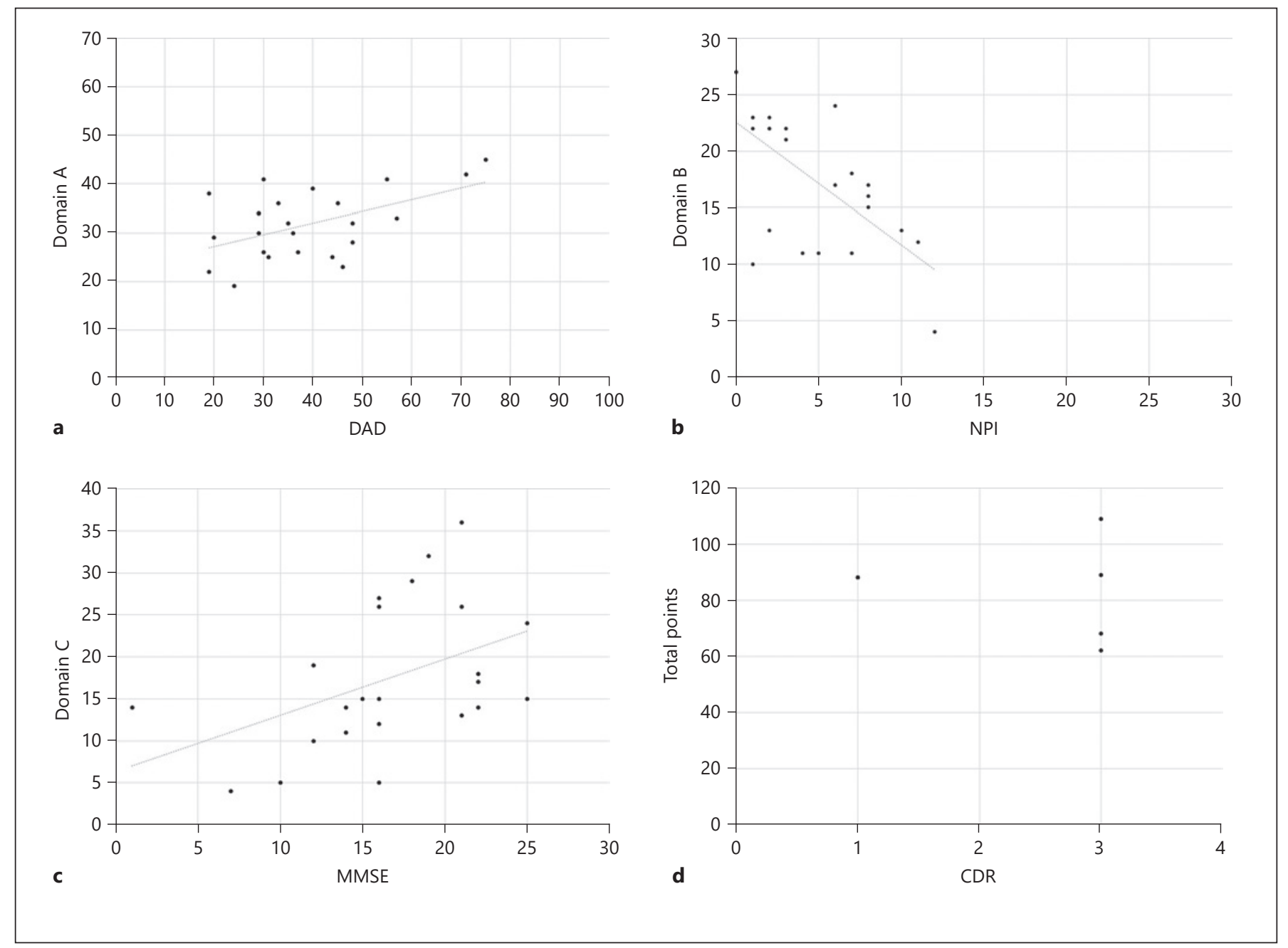

Fig. 3. The relationship between ABC-DS score and their respective reference neuropsychological instruments in patients with vascular dementia. The relationship between domain A of ABCDS and DAD (correlation coefficient: $\left.R^{2}=0.509\right)(\mathbf{a})$, the relationship between domain B of ABC-DS and NPI (correlation coefficient: $\left.R^{2}=0.646\right)(\mathbf{b})$, the relationship between domain $\mathrm{C}$ of $\mathrm{ABC}$ -

in patients with a global CDR score of 1 (mild dementia), suggesting the limited utility of the ABC-DS scale in assessing patients with mild dementia. These results confirmed the effectiveness of the ABC-DS scale in assessing patients with moderate-to-severe dementia. The majority of older patients with dementia who are visiting or residing in psychiatric hospitals and clinics in Japan have moderate or severe dementia [23]. This suggests the usefulness of the ABC-DS scale in geriatric clinical practice in Japan.

Moreover, we compared the results by the type of care provided to study participants (outpatient hospital vs.
DS and MMSE (correlation coefficient: $\left.R^{2}=0.44\right)(\mathbf{c})$, and the relationship between total scores of ABC-DS and CDR (correlation coefficient: $\left.R^{2}=0.476\right)($ d). ABC-DS, ABC Dementia Scale; DAD, Disability Assessment for Dementia; NPI, Neuropsychiatric Inventory; MMSE, Mini-Mental State Examination; CDR, Clinical Dementia Rating.

residential care facility). The 3 domains and the total score showed moderate or strong correlations with their respective reference tests for both types of institutions examined, supporting the scale's applicability to different care settings. Furthermore, our study yielded correlation coefficients similar to those reported in previous studies in outpatient and inpatient clinical settings [10]. The scale's wide-range applicability underscores its utility in Japan and other regions where home-based support and care for patients with dementia are advocated. One major drawback of this scale related to Question 10, which asks about the help the patient needs regarding medication. It 
Table 4. Correlation coefficients between the ABC-DS and respective reference tests among patients with vascular dementia

\begin{tabular}{lcccc}
\hline & Correlation & Lower & Upper & $\begin{array}{l}p \\
\text { value }\end{array}$ \\
& & & & \\
\hline Domain A versus DAD & & & & \\
CDR 1 (mild) & 0.815 & 0.329 & 0.960 & $<0.01$ \\
CDR 2 (moderate) & -0.077 & -0.647 & 0.548 & 0.822 \\
CDR 3 (severe) & 0.948 & $\mathrm{Na}^{*}$ & $\mathrm{na}^{*}$ & 0.207 \\
Domain B versus NPI & & & & \\
CDR 1 (mild) & -0.419 & -0.847 & 0.340 & 0.262 \\
CDR 2 (moderate) & -0.788 & -0.942 & -0.357 & $<0.01$ \\
CDR 3 (severe) & -0.217 & na* & $\mathrm{na}^{*}$ & 0.861 \\
Domain C versus MMSE & & & & \\
CDR 1 (mild) & 0.427 & -0.331 & 0.850 & 0.252 \\
CDR 2 (moderate) & 0.663 & 0.105 & 0.904 & $<0.05$ \\
CDR 3 (severe) & 0.196 & na* & $\mathrm{na}^{*}$ & 0.874 \\
\hline
\end{tabular}

ABC-DS, ABC Dementia Scale; DAD, Disability Assessment for Dementia; CDR, Clinical Dementia Rating; NPI, Neuropsychiatric Inventory; MMSE, Mini-Mental State Examination. * Could not be calculated because the sample size was 3 . is a common procedure at many residential dementia care facilities to hand-deliver medications to residents regardless of their mental and functional capabilities. As a result, their score on this question was a maximum of 3 points on a 1-9 scale. This suggests the need to revise the anchor points for this item.

The distribution of the different types of dementia in Japan was previously shown to be $67.6 \%$ for $\mathrm{AD}, 19.5 \%$ for $\mathrm{VaD}$, and $4.3 \%$ for $\mathrm{DLB}$, but in this study, it was $37.2 \%$ for $\mathrm{AD}, 9.8 \%$ for $\mathrm{VaD}$, and $5.8 \%$ for DLB (also, $11.7 \%$ for the combination of $\mathrm{AD}$ and $\mathrm{VaD}$, and $5.8 \%$ for the combination of $\mathrm{AD}$ and $\mathrm{DLB})$. In this research, 1 hospital in Nagasaki Prefecture and patients who use the facility that yielded the above distribution actively treat patients with BPSD. This may lead to bias compared to previous studies, as well as different frequencies of dementia types. However, we have been actively recruiting new patients at both institutions to reduce bias as much as possible.

We evaluated the correlation between the ABC-DS score and the global CDR score. In a previous study of the ABC-DS, scores of 13-70, 71-85, 86-100, and 101-117 corresponded to global CDR scores of 3, 2, 1, and 0.5-0, respectively [21]. In our study, ABC-DS scores of 13-58, 59-75, and 76-117 corresponded to global CDR scores of 3,2 , and $1,0.5-0$, respectively. The ABC-DS cutoff scores that differentiated global CDR scores of 3 and 2 and of 2 and 1 were 70.5 and 85.5 , respectively, in the previous
Table 5. Correlation coefficients between the ABC-DS and respective reference tests (type of care)

\begin{tabular}{|c|c|c|c|c|}
\hline & Correlation & Lower & Upper & $\begin{array}{l}p \\
\text { value }\end{array}$ \\
\hline \multicolumn{5}{|l|}{ Domain A versus DAD } \\
\hline Outpatient hospital & 0.843 & 0.766 & 0.896 & $<0.01$ \\
\hline Dementia facility & 0.793 & 0.549 & 0.912 & $<0.01$ \\
\hline \multicolumn{5}{|l|}{ Domain B versus NPI } \\
\hline Outpatient hospital & -0.792 & -0.861 & -0.694 & $<0.01$ \\
\hline Dementia facility & -0.663 & -0.851 & -0.324 & $<0.05$ \\
\hline \multicolumn{5}{|c|}{ Domain C versus MMSE } \\
\hline Outpatient hospital & 0.824 & 0.744 & -0.886 & $<0.01$ \\
\hline Dementia facility & 0.627 & 0.268 & 0.833 & $<0.05$ \\
\hline \multicolumn{5}{|l|}{ TDD versus CDR-SOB* } \\
\hline Outpatient hospital & -0.635 & -0.749 & -0.484 & $<0.01$ \\
\hline Dementia facility & -0.829 & -0.911 & -0.544 & $<0.01$ \\
\hline
\end{tabular}

ABC-DS, ABC Dementia Scale; DAD, Disability Assessment for Dementia; NPI, Neuropsychiatric Inventory; MMSE, MiniMental State Examination; CDR, Clinical Dementia Rating. * CDR-SOB: total sum of box scores in the CDR. study, and 58.5 and 76.0, respectively, in our study. Our cutoff scores were lower than those of the previous study, probably because our study included no participants with normal cognitive function and the study population consisted of both $\mathrm{AD}(n=38)$ and non-AD $(n=64)$ dementias. The predictive validity of the ABC-DS scale for dementia severity warrants further evaluation. Since the CDR-SOB score and TDD showed a closer correlation than the CDR-SOB* score and the total ABC-DS score (Table 2), we concluded that TDD was more useful than the total ABC-DS score.

We first assessed the ABC-DS scale at a dementia facility prior to outpatient care, but there were problems with the usefulness of the instrument. In particular, some ABC-DS scores could not be obtained at the dementia facility because the staff dispense patients' drugs. We therefore implemented the ABC-DS scale at an outpatient hospital and demonstrated that it was effective in this setting. Therefore, we compared the dementia facility with the outpatient hospital and found a similar relationship between the ABC-DS score and the CDR-SOB score at both institutions.

The cross-sectional nature of our study is its major limitation. The large intra-patient variability of BPSD underscores the importance of longitudinal studies [24,25]. Previous studies suggested that the ABC-DS scale is useful for tracing chronological changes in dementia sever- 
ity. We provided interventions for some patients with AD-type dementia in this study, and followed up all patients for 6 months, at which time the ABC-DS score changed before BPSD changed, and treatment of BPSD improved ABC-DS scores. Our future research will evaluate longitudinal score changes associated with medical interventions in patients with dementia.

\section{Acknowledgements}

The authors thank the participants and the staff at MinamiNagasaki Clinics and Group-Home Erufu.

\section{Statement of Ethics}

The research was conducted ethically in accordance with the World Medical Association Declaration of Helsinki, and Ethical Guidelines for Medical Research for Humans on Japanese Ministry of Health, Labor and Welfare and the Rules of the Ethics Committee of Nagasaki University Graduate School of Biomedical Sciences. This study was approved by the Ethics Committee of the
Nagasaki University Graduate School of Biomedical Sciences (No. 19071101). All patients or their families have agreed with written informed consent in the "Statement of Ethics."

\section{Conflict of Interest Statement}

There is no conflict of interest to declare.

\section{Funding Sources}

This research was supported by Management Grant of Nagasaki University.

\section{Author Contributions}

W.S. and K.S. designed the study, planned the statistical analyses, collected the data, performed statistical analyses, interpreted the results, and wrote the manuscript. J.M. and A.N. advised on the study design and contributed to data collection. All authors approved the final version of the manuscript for submission.

\section{References}

1 Umeda-Kameyama Y, Mori T, Wada-Isoe K, Kikuchi T, Kojima S, Kagimura T, et al. Development of a novel convenient Alzheimer's disease assessment scale, the $\mathrm{ABC}$ dementia scale, using item response theory. Geriatr Gerontol Int. 2019;19(1):18-23.

2 Kato S, Shimogaki H, Onodera A, Ueda H, Oikawa K. Development of the revised version of Hasegawa's dementia scale (HDS-R) (in Japanese). J Geriatr Psychiatry. 1991;2: 1339-47.

3 Mori T, Kikuchi T, Umeda-Kameyama Y, Wada-Isoe K, Kojima S, Kagimura T, et al. ABC dementia scale: a quick assessment tool for determining Alzheimer's disease severity. Dement Geriatr Cogn Dis Extra. 2018;8:8597.

4 Kim KW, Lee DY, Jhoo JH, Youn JC, Suh YJ, Jun YH, et al. Diagnostic accuracy of minimental status examination and revised Hasegawa dementia scale for Alzheimer's disease. Dement Geriatr Cogn Disord. 2005; 19(5-6):324-30.

5 Folstein MF, Folstein SE, McHugh PR. "Minimental state." a practical method for grading the cognitive state of patients for the clinician. J Psychiatr Res. 1975;12:189-98.

6 Gélinas I, Gauthier L, McIntyre M, Gauthier S. Development of a functional measure for persons with Alzheimer's disease: the disability assessment for dementia. Am J Occup Ther. 1999;53:471-81.
7 Cummings JL, Mega M, Gray K, Rosenberg-Thompson S, Carusi DA, Gornbein J. The neuropsychiatric inventory: comprehensive assessment of psychopathology in dementia. Neurology. 1994;44(12):230814.

8 Morris JC. The clinical dementia rating (CDR): current version and scoring rules. Neurology. 1993;43(11):2412-4.

9 Umeda-Kameyama Y, Mori T, Wada-Isoe K, Kikuchi T, Kojima S, Kagimura T, et al. Development of a novel convenient Alzheimer's disease assessment scale, the $\mathrm{ABC}$ dementia scale, using item response theory. Geriatr Gerontol Int. 2019;19(1):18-23.

10 Kikuchi T, Mori T, Wada-Isoe K, Umeda-Kameyama Y, Kagimura T, Kojima S, et al. A novel dementia scale for Alzheimer's disease. J Alzheimers Dis Parkinsonism. 2018;8:2.

11 Mori T, Kikuchi T, Umeda-Kameyama Y, Wada-Isoe K, Kojima S, Kagimura T, et al. $\mathrm{ABC}$ dementia scale: a quick assessment tool for determining Alzheimer's disease severity. Dement Geriatr Cogn Dis Extra. 2018;8:8597.

12 Rosenberg A, Ngandu T, Rusanen M, Antikainen R, Bäckman L, Havulinna S, et al. Multidomain lifestyle intervention benefits a large elderly population at risk for cognitive decline and dementia regardless of baseline characteristics: the FINGER trial. Alzheimers Dement. 2018;14(3):263-70.
13 Ngandu T, Lehtisalo J, Solomon A, Levälahti E, Ahtiluoto S, Antikainen R, et al. A 2 year multidomain intervention of diet, exercise, cognitive training, and vascular risk monitoring versus control to prevent cognitive decline in at-risk elderly people (FINGER): a randomised controlled trial. Lancet. 2015; 385(9984):2255-63.

14 American Psychiatric Association. Diagnostic and statistical manual of mental disorders. 4th, text revision. Washington: American Psychiatric Association; 2000.

15 McKhann GM, Knopman DS, Chertkow H, Hyman BT, Jack CR Jr, Kawas CH, et al. The diagnosis of dementia due to Alzheimer's disease: recommendations from the national institute on aging-Alzheimer's association workgroups on diagnostic guidelines for Alzheimer's disease. Alzheimers Dement. 2011;7:263-9.

16 McKhann G, Drachman D, Folstein M, Katzman R, Price D, Stadlan EM. Clinical diagnosis of Alzheimer's disease: report of the NINCDS-ADRDA work group under the auspices of department of health and human services task force on Alzheimer's disease. Neurology. 1984;34:939-44.

17 Albert MS, DeKosky ST, Dickson D, Dubois B, Feldman HH, Fox NC, et al. The diagnosis of mild cognitive impairment due to $\mathrm{Al}$ zheimer's disease: recommendations from the national institute on aging-Alzheimer's association workgroups on diagnostic guidelines for Alzheimer's disease. Alzheimers Dement. 2011;7:270-9. 
18 McKeith IG, Boeve BF, Dickson DW, Halliday G, Taylor JP, et al., Diagnosis and management of dementia with Lewy bodies: fourth consensus report of the DLB consortium. Neurology. 2017. 89(1):88-100.

19 Kikuchi T, Mori T, Isoe KW, Kameyama YU, Kagimura T, Kojima S, et al. A novel dementia scale for Alzheimer's disease. J Alzheimers Dis Parkinsonism. 2018;8:2.

20 Rouch I, Pongan E, Trombert B, Fabre F, Auguste N, Sellier C, et al. One-year evolution of behavioral and psychological symptoms of dementia in patients initially hospitalized in cognitive behavioral units: the EVITAL prospective cohort. J Alzheimers Dis. 2017;57(1): $147-55$.
21 Nunes PV, Schwarzer MC, Leite REP, Ferretti-Rebustini REL, Pasqualucci CA, Nitrini R, et al. Neuropsychiatric inventory in community-dwelling older adults with mild cognitive impairment and dementia. J Alzheimers Dis. 2019;68(2):669-78.

22 Makimoto K, Kang Y, Kobayashi S, Liao XY, Panuthai S, Sung HC, et al. Prevalence of behavioural and psychological symptoms of dementia in cognitively impaired elderly residents of long-term care facilities in East Asia: a cross-sectional study. Psychogeriatrics. 2019;19(2):171-18.
23 Kivipelto M, Solomon A, Ahtiluoto S, Ngandu T, Lehtisalo J, Antikainen R, et al. The Finnish geriatric intervention study to prevent cognitive impairment and disability (FINGER): study design and progress. Alzheimers Dement. 2013;9(6): 657-65.

24 Nakanishi M, Miyamoto Y, Nakashima T, Shindo Y, Nishida A. Care preferences of healthy, middle-aged adults in Japan and the USA if they acquired dementia: a cross-sectional observational study. Geriatr Gerontol Int. 2019;19(8):829-33.

25 Simon V, Csiba L, Kardos L, Frecska E, Hortobágyi T. Behavioural and psychological symptoms in neurocognitive disorders: specific patterns in dementia subtypes. Open Med. 2019;14:307-16. 